\title{
Powering an Engine with Quantum Coherence
}

\section{Experiments demonstrate a quantum-coherence-induced power increase for quantum heat engines over their classical counterparts.}

\section{by Janet Anders*}

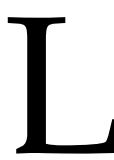

ook out of the window of a train traveling at 180 $\mathrm{mph}$, and you get an immediate sense of the power of modern engines. Through improved understanding of the laws of thermodynamics, and plenty of engineering ingenuity, these machines have come a long way since 1829 when Stephenson's Rocket-an early steam train-set the record for the fastest locomotive, achieving a speed of $30 \mathrm{mph}$. An entirely different avenue exists to increase an engine's power but for microscopic scale engines made of atoms rather than macroscopic train engines [1]. For tiny engines that operate in the quantum regime, researchers have predicted a quantum boost to engine ef-
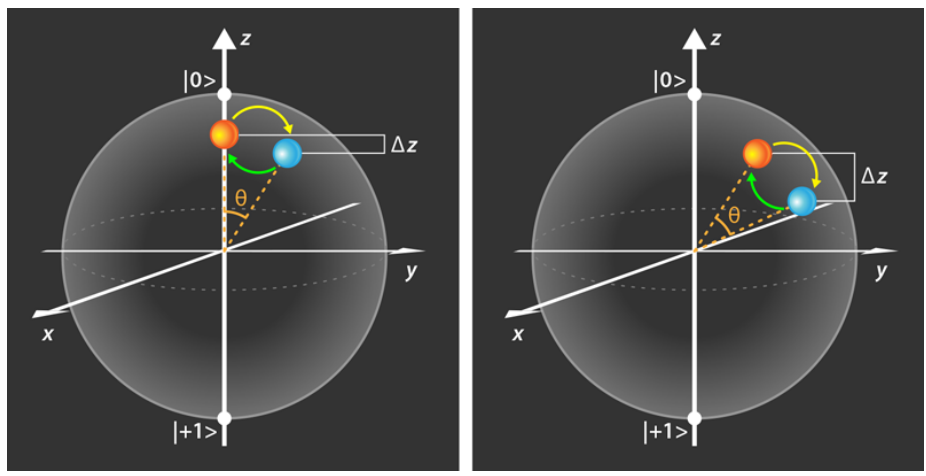

Figure 1: Sketch showing the cycle of the quantum engine realized with a nitrogen-vacancy (NV) center. The top sphere (orange) in each image indicates the state of the NV center at the start of each cycle, which is either classical (left) or quantum (right), depending on whether the initial state is quantum coherent. During the work stroke (yellow arrow) the NV center loses energy, indicated by $\Delta z$. The lost energy is drawn from the NV center as work. A green microwave pulse (green arrow) is then applied to the center, connecting it to two thermal baths, an action that restores the initial state. The cycle then begins again. (APS/Alan Stonebraker)

\footnotetext{
*Department of Physics and Astronomy, University of Exeter, Ex-
} eter, United Kingdom ficiency [2]. Using an ensemble of nitrogen-vacancy (NV) centers in diamond to realize a quantum heat engine, James Klatzow from the University of Oxford in the UK and his colleagues have now measured this quantum power increase for the first time [3].

Classical heat engines generate power by performing a series of "strokes" that convert thermal energy (heat) into mechanical energy (work). Quantum heat engines operate in a similar way. But in contrast to their classical counterparts, the energetic states of a quantum engine's so-called working fluid, which acts like the steam in a steam engine, can be in a coherent superposition. This possibility led researchers to ask whether quantum engines could perform better than classical ones.

In 2015, Raam Uzdin from the Hebrew University of Jerusalem and colleagues answered this question, predicting a power increase for quantum engines that run in the socalled small-action limit [2]. In this limit, engine strokes are short, leading to small heat and work exchanges, and quantum coherence between different energy states of the engine becomes more prominent. Uzdin and colleagues suggested that this coherence leads to a boost in power for a quantum engine over a comparable classical counterpart. With the predictions standing, the challenge was set to demonstrate this quantum advantage experimentally.

The experiments performed by Klatzow and his colleagues meet this challenge [3]. In the team's setup, the two lowest energy levels of the NV center, $|0\rangle$ and $|+1\rangle$, provide the two levels of a qubit and act as the working fluid. Higher energy levels play the roles of two thermal baths with different temperatures. Placing the NV centers in a magnetic field, the researchers reversed the energetic ordering of the $|0\rangle$ and $|+1\rangle$ energy levels, creating an initial state with a "population inversion." In addition, this state could host quantum coherence-meaning there was a fixed relationship between the probability amplitudes for the two qubit levels. The team realized a work stroke by applying a microwave pulse that rotated the qubit by an angle $\theta$, which is equivalent to adjusting amplitudes (Fig. 1). This rotation lowered the NV center's energy by an amount $\Delta z$ and this energy was extracted as work. Finally, the team hit the centers with a green laser that coupled the qubit levels to the thermal baths and 
ultimately caused the centers to return to the initial qubit state. This two-stroke cycle was repeated more than 100,000 times, for cycles lasting between 30 and $180 \mathrm{~ns}$.

For small $\theta$-the small-action limit-Klatzow and colleagues found that the power output of their engine with quantum coherence was significantly higher than that calculated for the same engine with no initial coherence. This increase arises because of a larger value of $\Delta \mathrm{z}$ (Fig. 1).

These experiments represent the first demonstration of a quantum enhancement in a thermodynamic heat engine, a seismic achievement, comparable to the first transmission of a cryptographic key using quantum encoding and to the first factorization of a number using Shor's algorithm. It should be noted, however, that the team only infers the engine's power output indirectly through measurements of stimulated emission from the NV centers. Direct measurements of the work drawn from a quantum engine are, in principle, possible, as demonstrated recently in a quantum Maxwell's demon experiment realized with a superconducting qubit [4]. But the small rotation angles in the NV center engine give only very tiny work outputs, which make direct work measurements unfeasible.

While the findings reported here agree with the 2015 predictions of Ref. [2], their relation to other recent theoretical predictions can be confusing. A related 2016 study showed that quantum coherence can be used up like a "fuel" to draw work [5]. The study implied that recreating coherence required the same work as that drawn when using coherence up, indicating a null cycle. But that analysis included only a single thermal bath, while the NV-center engine couples to two baths at two different temperatures. In the Klatzow engine, coherence is created and then reduced, but it is never completely removed. Overall, this operation leads to a net work output for the quantum engine that is larger than that of a comparable classical engine. It is also worth noting that since Klatzow and colleagues use two thermal baths, their system differs fundamentally from that modeled in a 2014 study predicting "catalytic coherence" - the ability to use coherence and a single thermal bath to cyclically draw work with no degradation. That prediction breaks the second law of thermodynamics, and it was based on an argument that failed to account for correlations between repeated iterations of the engine's cycle [6].

While the work of Klatzow and colleagues is truly groundbreaking, there is reason to treat the results with caution and to not yet declare that the role of coherence in quantum heat engines is settled. The team only produced a single data point in the regime excluded by classical physics, and thus more research is still needed to fully uncover the significance of quantum coherence for thermodynamics. We can expect a flurry of new experiments that attempt to provide measurements of quantum thermodynamics signatures, both in established quantum platforms, such as superconducting qubits [4] and trapped atoms [1], and in new and future platforms that include optomechanical setups with suspended nanotubes [7], electromechanical engines [8], and optical nanoengines made from levitated nanoparticles [9]. For now, these sophisticated experiments will foremost serve to explore fundamental physics. But who knows, in two centuries, we may look back at this time as the birth of the quantum coherent engine.

This research is published in Physical Review Letters.

\section{REFERENCES}

[1] J. Roßnagel, S. T. Dawkins, K. N. Tolazzi, O. Abah, E. Lutz, F. Schmidt-Kaler, and K. Singer, "A single-atom heat engine," Science 352, 325 (2016).

[2] R. Uzdin, A. Levy, and R. Kosloff, "Equivalence of quantum heat machines, and quantum-thermodynamic signatures," Phys. Rev. X 5, 031044 (2015).

[3] J. Klatzow, J. N. Becker, P. M. Ledingham, C. Weinzetl, K. T. Kaczmarek, D. J. Saunders, J. Nunn, I. A. Walmsley, R. Uzdin, and E. Poem, "Experimental demonstration of quantum effects in the operation of microscopic heat engines," Phys. Rev. Lett. 122, 110601 (2019).

[4] N. Cottet, S. Jezouin, L. Bretheau, P. Campagne-lbarcq, Q. Ficheux, J. Anders, A. Auffèves, R. Azouit, P. Rouchon, and B. Huard, "Observing a quantum Maxwell demon at work," Proc. Natl. Acad. Sci. 114, 7561 (2017).

[5] P. Kammerlander and J. Anders, "Coherence and measurement in quantum thermodynamics," Sci. Rep. 6, 22174 (2016).

[6] J. Vaccaro, S. Croke, and S. Barnett, "Is coherence catalytic?" J. Phys. A 51, 414008 (2018).

[7] N. Ares, T. Pei, A. Mavalankar, M. Mergenthaler, J. H. Warner, G. A. D. Briggs, and E. A. Laird, "Resonant optomechanics with a vibrating carbon nanotube and a radio-frequency cavity," Phys. Rev. Lett. 117, 170801 (2016).

[8] D. Goldwater, B. Stickler, L. Martinetz, T. E. Northup, K. Hornberger, and J. Millen, "Levitated electromechanics: All-electrical cooling of charged nano- and micro-particles," Quantum Sci. Technol. 4, 024003 (2019).

[9] A. Dechant, N. Kiesel, and E. Lutz, "All-optical nanomechanical heat engine," Phys. Rev. Lett. 114, 183602 (2015).

10.1103/Physics.12.32 